\title{
Acute posterior thigh pain in an athlete
}

\author{
Timothy Dickson ${ }^{1}$ (D) - George Koulouris ${ }^{1}$
}

Published online: 14 October 2016

(C) ISS 2016

\begin{abstract}
Answer
Diagnosis: grade I myofascial strain of an accessory muscular slip and adjoining muscle fibres, connecting an accessory tensor fascia suralis (TFS) muscle and the distal biceps femoris.
\end{abstract}

\section{Discussion}

Magnetic resonance images of the left thigh in this patient following an acute running injury demonstrate a grade I strain involving a slip of the muscle connecting an accessory hamstring muscle, known as the tensor fascia suralis (TFS). The strain is located along the lateral aspect of the TFS, and along the medial myofascial boundary of the long head of the biceps femoris muscle. Distally, the TFS continues as a thin tendon that inserts onto the superficial fascia overlying the medial head of the gastrocnemius muscle (as shown in Fig. 3 of the Question). The most distal extent in this case was not completely imaged. The patient was conservatively managed and returned to sport without any need for surgical intervention.

The TFS is an accessory muscle of the posterior thigh and was first described in 1813 [1]. It can arise from the distal aspect of any of the hamstring muscles, most commonly from the distal semitendinosus muscle [2], followed by the long head of the biceps femoris [3]. It passes posterior to the medial

The case presentation can be found at doi: 10.1007/s00256-016-2499-z

Timothy Dickson

timd@melbourneradiology.com.au

1 Melbourne Radiology Clinic, 3-6/100 Victoria Pde, East Melbourne, VIC 3002, Australia gastrocnemius muscle [4] and has a variable insertion onto the sural fascia, the medial head of the gastrocnemius, or the superficial aspect of the Achilles tendon [5]. The innervation of this muscle is via the tibial component of the sciatic nerve and although no biomechanical studies have been reported, its anatomical location (and name) presumes that it tenses the sural fascia of the posterior leg [5], assisting in knee flexion.

The description of TFS is sporadic in the literature, with the majority of case reports describing unilaterality with the exception of a recent dissection study [3]. Its first description in the radiological literature [6] describes its appearances on MR imaging as a cylindrically shaped muscle in the superficial aspect of the popliteal fossa, posterior to the medial head of the gastrocnemius muscle, with signal characteristics typical of skeletal muscle, also noted in subsequent case reports [7, 8].

As the TFS is located superficially, it can present clinically as a subcutaneous mass [4] and similar to other accessory muscles, it may enlarge and become more apparent upon active contraction. Therefore, it may present as a mass [6] and potentially lead to unnecessary imaging, biopsy and/or surgical intervention. The sural nerve and the short saphenous vein are immediately lateral in relation to the TFS [3]. Cadaveric entrapment of the lateral sural cutaneous nerve has been described [9]; however, this has yet to be described clinically. The relationship between the TFS and its origin from either the semitendinosus or long head of the biceps femoris muscles and variably connected muscle fibres may predispose it to injury at the myofascial interface, which has been previously described as a potential mechanism of injury occurring between the short and long heads of the biceps femoris muscles at their corresponding myofascial interface [10].

The characteristic normal muscle fibres and distal aponeurotic tendon of the TFS, whether evaluated with MRI or ultrasound, should alert the radiologist to the presence of an accessory muscle. As with all accessory muscles, correct 
identification is of prime importance, to avoid confusion with a mass that could potentially lead to unnecessary investigations and intervention.

\section{Compliance with ethical standards}

Conflicts of interest The authors declare that they have no conflicts of interest.

\section{References}

1. Kelch WG. Abweichung des Biceps Femoris. Beitr Pathologischen Anat. 1813;8:42.

2. Luca C, Stan C, Popescu D, Mota O, Popescu P, Davalciuc O. The tensor fasciae suralis muscle - case report. Revista Romana de Anatomie Functionala si Clinica, Macro-si Microscopica si de Antropologie 2009;8:501-3.
3. Gandhi KR, Wabale RN, Farooqui MS. Bilateral presentation of tensor fascia suralis muscle in a male cadaver: a case report. Int J Anat Res. 2015;3(4):1745-8.

4. Duc SR et al. First report of an accessory popliteal muscle: detection with MRI. Skeletal Radiol. 2004;33(7):429-31.

5. Tubbs RS, Salter EG, Oakes WJ. Dissection of a rare accessory muscle of the leg: the tensor fasciae suralis muscle. Clin Anat. 2006;19(6):571-2.

6. Chason DP, Schultz SM, Fleckenstein JL. Tensor fasciae suralis: depiction on MR images. AJR Am J Roentgenol. 1995;165(5): $1220-1$.

7. Montet $\mathrm{X}$ et al. Sonographic and MRI appearance of tensor fasciae suralis muscle, an uncommon cause of popliteal swelling. Skeletal Radiol. 2002;31(9):536-8.

8. Kim KH et al. MR imaging and ultrasonographic findings of tensor fasciae suralis muscle: a case report. J Korean Soc Radiol. 2015;73(4):249-51.

9. Amruth M, Sagorika M, Balakrishna AG. Entrapment of sural nerve. Anat Karnataka. 2008;3(2):63-6.

10. Koulouris G, Connell D. Hamstring muscle complex: an imaging review. Radiographics. 2005;25(3):571-86. 